\title{
Testing emissions equivalency metrics against climate policy goals
}

\author{
Morgan R. Edwards ${ }^{\mathrm{c}}$, James McNerney ${ }^{\mathrm{c}}$, Jessika E. Trancik ${ }^{\mathrm{c}, \mathrm{d}, *}$ \\ ${ }^{c}$ Institute for Data, Systems, and Society, Massachusetts Institute of Technology, 77 Massachusetts Avenue, Cambridge, MA \\ 02139, USA \\ ${ }^{d}$ Santa Fe Institute, 1399 Hyde Park Rd, Santa Fe, NM 87501, USA
}

\begin{abstract}
The climate impacts of energy technologies are frequently assessed using equivalency metrics, which convert emissions of multiple greenhouse gases to a common scale. Numerous metrics have been proposed that incorporate, in different ways, information about the time-dependent impacts of gases. However, more focus has been placed on proposing metrics than on testing their performance in real-world use cases. Here we present a testing approach that simulates how metrics would affect the selection of energy technology portfolios that comply with a $\mathrm{CO}_{2}$-equivalent emissions cap. Unintended radiative forcing outcomes can occur, emphasizing the need to test metrics in a practical context. We demonstrate the approach for policies designed to limit radiative forcing and discuss extensions to limits on temperature or economic impacts. Metric performance is evaluated by (i) how much actual radiative forcing overshoots the intended stabilization level, and (ii) the level of energy consumption permitted. We use this testing approach to study a variety of metrics based on an estimated radiative forcing stabilization time under two climate policy goals. We find that these goal-inspired metrics, if chosen carefully, can exhibit performance improvements over the standard global warming potential (GWP) while maintaining its transparency and ease-of-use. These alternative metrics can significantly reduce the overshoot in radiative forcing observed with the GWP, at a small cost in energy consumption. Moreover, simple metrics can exhibit similar performance improvements to more complex ones.
\end{abstract}

Keywords. Emissions metrics, climate change, energy technology evaluation.

\section{Introduction}

A method for comparing greenhouse gases is necessary to evaluate the climate impacts of energy technologies, which emit multiple greenhouse gases during their life cycles $[4,7,9,10,16,17,20,53]$, on a single scale. Similarly, policies regulating greenhouse gases require information on the relative climate impacts of emissions. However, gases have different atmospheric lifetimes [41], which makes these comparisons challenging $[30,31,37]$. There is no single objective measure that converts the climate impact of one gas into an equivalent amount of another gas perfectly in all situations [18]. One common approach to comparing

(C) 2016. This manuscript version is made available under the Elsevier user license

http://www.elsevier.com/open-access/userlicense/1.0/ 

equivalent mass units $[10,13,16,19,20]$. The equivalency metric approach enables emissions to be assessed by distributed decision makers and regulated against a 'single-basket' $\mathrm{CO}_{2}$-equivalent budget.

Other approaches have also been proposed for comparing technologies. One example is the technology warming potential (TWP), which is designed to facilitate pairwise comparisons of technologies [4, 9, 21]. The TWP compares how impacts decay over time following the use of two different technologies, allowing users to examine impacts at multiple time horizons of interest. A similar approach can be used to compare the climate impacts of end-use sector emissions [17]. Because these methods do not propose a particular time horizon for technology or end-use sector comparisons, they are not designed to provide an assessment of emissions against a $\mathrm{CO}_{2}$-equivalent policy.

Some have also pointed out the pitfalls of single-scale comparisons of gases using equivalency metrics and have suggested that emissions of gases with dissimilar lifetimes should be regulated under separate 'baskets' $[11,40]$. However, this approach would still require a comparison of gases to set the limits for each basket, and some means of assessing the actions of distributed decision makers against a central climate policy goal. Additionally, the inability to trade across different baskets means less flexibility in how emissions reductions are met, potentially raising costs [51, 52].

Despite the inherent difficulties in comparing greenhouse gases using equivalency metrics, these metrics continue to see widespread use in technology evaluation [13]. Current climate policies are also predominantly framed in $\mathrm{CO}_{2}$-equivalent terms $[1,3,45]$. This is likely because they allow distributed actors to assess activities resulting in emissions of several gases against a central goal, and because $\mathrm{CO}_{2}$-equivalent policies offer more flexibility in emissions reductions. Consequently, it is important to understand when equivalency metrics provide adequate comparisons of gases and when metrics are misleading or inappropriate, by testing their performance in simulations that represent how they would be used in the real world to make technology and policy decisions.

The most common equivalency metric, the global warming potential (GWP), compares emitted gases based on their integrated radiative forcing contributions from the time of emission over a fixed duration, typically 100 years [22]. Numerous alternative metrics have been proposed [14, 24, 26, 28, 32, 33, 39, 38, 43], although none has supplanted the GWP [37]. Some of these alternatives maintain the basic approach of the GWP, proposing a constant exchange rate between gases based on temperature changes $[32,39,43]$ or economic impacts [26, 33], either a fixed number of years into the future [39] or integrated over a specified duration [32], with economic metrics often incorporating discounting [26, 33]. The fixed time variable in these static metrics, either the duration of integration or number of years in the future when impacts are evaluated, strongly determines metric values but is often arbitrarily chosen [5]. Static metrics may also be based on a particular time of interest, such as the timing of radiative forcing or temperature stabilization.

Other metric proposals depart more fundamentally from the static GWP and allow the exchange rate between gases to depend on the time of emission, and therefore to change over time. The motivation for 

broader policy goals, such as minimizing the costs of climate change or avoiding dangerous interference with the climate system. Some dynamic metrics, which we call complex dynamic metrics, are based on the costs and benefits [29] or (more typically) the cost-effectiveness [28] of mitigating each kind of gas, given a specified climate and economic scenario. Other simple dynamic metrics have also been proposed, which require only information on physical variables [14,38], and possibly a discount rate [24], and may be more transparent for evaluating energy technologies and designing mitigation policies. Simple dynamic and static metrics anchored to an expected stabilization time both rely on similar inputs, but the former provide an emission-time-dependent comparison of gases.

However, neither static nor dynamic metrics have been systematically tested to see how they perform in realistic use cases, or compared with the standard GWP. The criteria for good performance has also not been explicitly defined [30]. Previous efforts to evaluate metrics have focused on calculating metric values under specific conditions [6, 42], or using theory to relate different metric formulations to one another conceptually $[12,44]$. Several studies have examined the economic performance of metrics assuming that climate constraints are obeyed $[8,15,25,34,49]$. However, this approach prevents the possibility that actual climate impacts may deviate from intended ones, by artificially capping climate outcomes (which, unlike emissions, cannot be directly controlled). A few studies consider only static metric performance but are limited to a single scenario [23] or extreme scenarios where all $\mathrm{CO}_{2}$ emissions are replaced with an equivalent amount of $\mathrm{CH}_{4}$ [11]. The latter approach provides a bound based on the worst case but does not account for the fact that greenhouse gases are co-emitted by activities, such as energy technology use, and cannot be completely substituted for one another.

Testing equivalency metrics in practical settings is critical. Metrics can fail to represent climate impacts as intended when they are put into actual use, despite possible theoretical arguments that may favor a metric. Here we test the performance of metrics in a realistic context in which the overall climate goal is known, but the exact emissions scenario for achieving that goal is unknown. The approach we describe is general and involves simulating the use of a metric in conjunction with an emission policy to determine allowable technology use, and calculating the resulting climate impacts and energy consumption permitted. This testing approach does not cap the realized climate outcome, as has been done in previously published work $[8,15,25,34,49]$, thereby capturing the real-world possibility of unintended consequences. We apply the approach to evaluate a range of radiative forcing metrics anchored to an expected radiative forcing stabilization time but of varying complexity. Our results suggest that these metrics can improve on the GWP when selecting energy technologies to meet commonly-cited climate mitigation goals. Simple metrics can also perform as well as more complex ones, suggesting a pragmatic approach be taken in metric selection. 


\section{Model}

Our approach to testing equivalency metrics consists of four steps: (1) Set a climate policy target. (2) Construct a $\mathrm{CO}_{2}$ emissions budget to meet the target. (3) Simulate energy technology choices that satisfy the emissions policy, using the metric to be tested to convert the multi-gas emissions of technologies to their $\mathrm{CO}_{2}$-equivalent values. (4) Compare the climate outcomes of this technology use scenario to the policy target. The model presented here is simple and illustrates the advantages of testing metrics and defining explicit criteria for metric performance under uncertainty. We use radiative forcing as the indicator of climate impact and a simple optimization model to select the consumption levels of two energy technology portfolios. Metric performance is evaluated by the overshoot of an intended radiative forcing stabilization level and the energy consumption allowed by the technology use scenario. The approach can be adapted to a variety of indicators (temperature impacts, economic impacts), technology selection methods (general equilibrium models, detailed sector-specific models), and performance measures.

We begin by generating a set of $\mathrm{CO}_{2}$ emissions budgets aimed at achieving a given radiative forcing stabilization goal. Budgeted emissions are modeled as $\mathrm{CO}_{2}$, but changing concentrations of $\mathrm{CH}_{4}$ and $\mathrm{N}_{2} \mathrm{O}$ due to previous emissions are also included. Emissions grow and decline according to a changing exponential growth rate [2] that begins at the present rate and, after the onset of mitigation, begins to decrease. Using this approach we study a range of stabilization scenarios varying from gradual mitigation to delay followed by rapid mitigation (Sec. A1). The range is not comprehensive of all stabilization scenarios but does capture those scenarios with the highest risk, which we will argue later are those with early stabilization dates.

Emissions are translated into concentrations using impulse response functions (Sec. A2), and concentrations determine radiative forcing using radiative efficiency values calculated for present-day concentrations (Sec. A3). Because mitigation scenarios typically involve an increase in $\mathrm{CO}_{2}$ concentrations and eventual decrease in $\mathrm{CH}_{4}$ concentrations relative to present day [50], using radiative efficiency values at present-day concentrations to test metrics will tend to underestimate the effects of $\mathrm{CH}_{4}$-induced overshoots (Sec. A3). However, this approach allows us to test metrics using the same removal rates and radiative efficiencies as in metric calculation, thereby isolating errors due to metric formulation, which are the focus of this study, from errors due to uncertainties in the physical parameters (which can be large [41]).

We present results for a simple case where the energy sector is supplied by two technology portfolios: one with the $\mathrm{CO}_{2}$ and $\mathrm{CH}_{4}$ intensities of the present-day energy sector and another with a $40 \%$ lower $\mathrm{CO}_{2}$ intensity but correspondingly higher $\mathrm{CH}_{4}$ intensity (Sec. B1). The mixed emissions of these technology portfolios are converted to $\mathrm{CO}_{2}$-equivalent units using the metric to be tested (Sec. B2). Actors in our simple model select a single portfolio in each year with the lowest $\mathrm{CO}_{2}$-equivalent emissions per unit energy provided. The portfolio remains the same over time if a static metric is used but may change if the metric is dynamic. Radiative forcing outcomes do not depend on absolute emissions intensities, meaning that these results also apply for low-carbon technologies with similar relative $\mathrm{CO}_{2}$ and $\mathrm{CH}_{4}$ intensities but may change under changing relative emissions intensities (Sec. B1). We then compute energy consumption and resulting 
multi-gas emissions, concentrations, and radiative forcing.

We test five metrics (Fig. 1), representing a range of formulations discussed in the literature [14, 24, 28, 38], calculated using radiative forcing as the indicator of impact. The instantaneous climate impact (ICI) compares gases based on their impact in an expected stabilization year $\left(t_{S}\right)$, while the cumulative climate impact (CCI) compares gases based on their summed impacts in all years up to and including $t_{S}$. These are examples of simple dynamic metrics. The optimized climate impact (OCI) is a complex dynamic metric and uses the Lagrange multiplier from a technology optimization model with a radiative forcing pathway constraint to weight impacts in each year up to $t_{S}$ (Sec. C). We also test a static metric, the stabilization warming potential (SWP), which takes the same form as the GWP but calculates the time horizon $\tau$ as the difference between $t_{S}$ and the present day. We compare these metrics to the standard GWP with a $\tau$ of 100 years.

The metrics are described by the formulas

$$
\begin{aligned}
\operatorname{GWP}(\tau) & =\frac{A_{\mathrm{M}} \int_{0}^{\tau} f_{\mathrm{M}}\left(t^{\prime \prime}, 0\right) d t^{\prime \prime}}{A_{\mathrm{K}} \int_{0}^{\tau} f_{\mathrm{K}}\left(t^{\prime \prime}, 0\right) d t^{\prime \prime}} & \\
\operatorname{SWP}\left(t_{\mathrm{S}}\right) & =\frac{A_{\mathrm{M}} \int_{0}^{t_{\mathrm{S}}-t_{0}} f_{\mathrm{M}}\left(t^{\prime \prime}, 0\right) d t^{\prime \prime}}{A_{\mathrm{K}} \int_{0}^{t_{\mathrm{S}}-t_{0}} f_{\mathrm{K}}\left(t^{\prime \prime}, 0\right) d t^{\prime \prime}} & t \leq t_{S} \\
\operatorname{ICI}\left(t^{\prime}, t_{\mathrm{S}}\right) & =\frac{A_{\mathrm{M}} f_{\mathrm{M}}\left(t_{\mathrm{S}}, t^{\prime}\right)}{A_{\mathrm{K}} f_{\mathrm{K}}\left(t_{\mathrm{S}}, t^{\prime}\right)} & t \leq t_{S} \\
\operatorname{CCI}\left(t^{\prime}, t_{\mathrm{S}}\right) & =\frac{A_{\mathrm{M}} \int_{t^{\prime}}^{t_{\mathrm{S}}} f_{\mathrm{M}}\left(t^{\prime \prime}, t^{\prime}\right) d t^{\prime \prime}}{A_{\mathrm{K}} \int_{t^{\prime}}^{t_{\mathrm{S}}} f_{\mathrm{K}}\left(t^{\prime \prime}, t^{\prime}\right) d t^{\prime \prime}} & t \leq t_{S}, \\
\mathrm{OCI}\left(t^{\prime}, t_{\mathrm{S}}, \lambda\right) & =\frac{A_{\mathrm{M}} \int_{t^{\prime}}^{t_{\mathrm{S}}} \lambda\left(t^{\prime \prime}\right) f_{\mathrm{M}}\left(t^{\prime \prime}, t^{\prime}\right) d t^{\prime \prime}}{A_{\mathrm{K}} \int_{t^{\prime}}^{t_{\mathrm{S}}} \lambda\left(t^{\prime \prime}\right) f_{\mathrm{K}}\left(t^{\prime \prime}, t^{\prime}\right) d t^{\prime \prime}} &
\end{aligned}
$$

where subscript $\mathrm{M}$ denotes $\mathrm{CH}_{4}$, subscript $\mathrm{K}$ denotes $\mathrm{CO}_{2}, A$ is the radiative efficiency, $f\left(t, t^{\prime}\right)$ gives the fraction of gas emitted at time $t^{\prime}$ remaining in the atmosphere at time $t, t_{0}$ is the present day, and $\lambda\left(t^{\prime \prime}\right)$ is a Lagrange multiplier. For all years after $t_{S}$, the dynamic metrics have values equal to the instantaneous tradeoff $A_{M} / A_{K}$.

Three out of the five metrics produce conversion factors that change depending on when emissions occur relative to an expected stabilization year (CCI, ICI, OCI). They are dynamic with respect to emissions timing. One out of the five metrics is based on the distance from the present day to the expected stabilization year (SWP). The SWP depends on the stabilization date, but once this date is chosen it is static with respect to emissions timing (Sec. A1). The GWP is also static and typically based on a single, somewhat arbitrarilyselected time horizon of 100 years $[5,22] .^{1}$

Tests of these metrics under uncertainty simulate the following scenario. Suppose a metric is agreed upon,

\footnotetext{
${ }^{1}$ The TWP takes the same inputs as an equivalency metric and produces multiple evaluations of the impacts of technology use over a range of time horizons. An equivalency metric, in contrast, produces a single comparison for a given time of use. A similar technology comparison can be reached using either approach if the TWP is supplemented by a chosen time horizon that is the same as that used in the corresponding equivalency metric; for example, a time horizon of 100 years would give the same comparison as the GWP [4].
} 

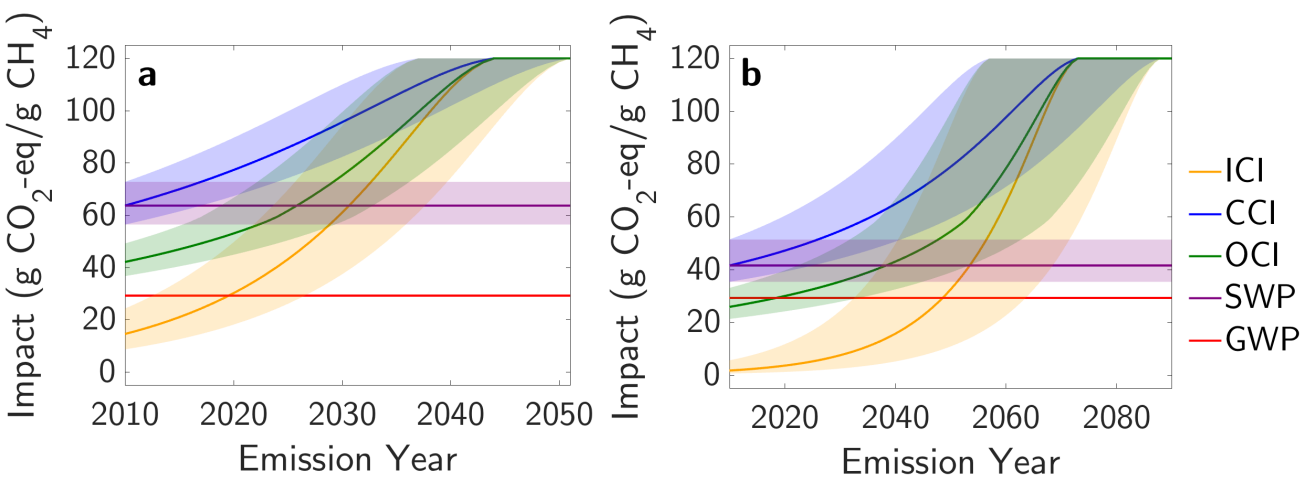

Figure 1: Metric values under a $3 \mathrm{Wm}^{-2}(\mathbf{a})$ and $4.5 \mathrm{Wm}^{-2}$ (b) stabilization target. Note the different scales on the horizontal axes. Shaded areas show the range of equivalency metric values for all calculated stabilization years (2037-2051 for 3 Wm ${ }^{-2}$ scenarios or 2057-2088 for $4.5 \mathrm{Wm}^{-2}$ scenarios). Solid lines indicate metric values using the midpoints of the full ranges (2044 and 2073) for $t_{S}$. Among the dynamic metrics, the ICI places the lowest impact value on $\mathrm{CH}_{4}$ early on, followed by the OCI and CCI. The SWP takes the same value as the CCI in the initial year and remains constant over the entire period. The GWP uses a constant value of 29 grams $\mathrm{CO}_{2}$-eq per gram $\mathrm{CH}_{4}$ in all years.

possibly using some information about the future scenario intended by policy. The metric is maintained over time, for example to provide a stable environment for technology research, development, and investment. (Note that while the GWP is periodically updated in the scientific literature [41], climate policies do not always use up-to-date values [1].) Since the future is inherently uncertain, emissions budgets that are eventually realized may differ from the ones assumed in calculating the equivalency metric. We use this scenario to test the performance of the GWP, as well as the SWP, ICI, CCI, and OCI using a best-guess stabilization time at the midpoint of the considered range. If the chosen metric is to be useful for technology planning and policy, it should perform well under perfect certainty, as well as show a degree of robustness to uncertainty in, for instance, the exact year when stabilization occurs.

\section{Results}

We test the performance of the GWP and the best-guess SWP, ICI, CCI, and OCI under radiative forcing stabilization targets of $3 \mathrm{Wm}^{-2}$ and $4.5 \mathrm{Wm}^{-2}$, which in equilibrium are roughly associated with $2^{\circ} \mathrm{C}$ and $3^{\circ} \mathrm{C}$ temperature change (recognizing the large range in climate sensitivity estimates [35]). Radiative forcing outcomes are shown in Figs. 2 and 3. Importantly, no metric perfectly reproduces the radiative forcing pathway from $\mathrm{CO}_{2}$ budget scenario. Deviations from this scenario depend on the amount of the emissions budget converted to $\mathrm{CH}_{4}$. The decay dynamics of $\mathrm{CH}_{4}$ differ from those of the hypothetical, budgeted $\mathrm{CO}_{2}$ emissions that $\mathrm{CH}_{4}$ is replacing, leading to an imperfect replacement and a different radiative forcing pathway from the one intended. The lower the impact value assigned to $\mathrm{CH}_{4}$, the more $\mathrm{CH}_{4}$ is emitted, and the larger the risk of a radiative forcing overshoot. However an overshoot is not inevitable, and the amount of overshoot differs across metrics.

For a $3 \mathrm{Wm}^{-2}$ target level, where the calculated stabilization time is around mid-century, the alternative metrics all outperform the GWP from a radiative forcing overshoot perspective. The ICI leads to a lower (though still large) and more temporary overshoot, while the OCI and SWP lead to very small or no 

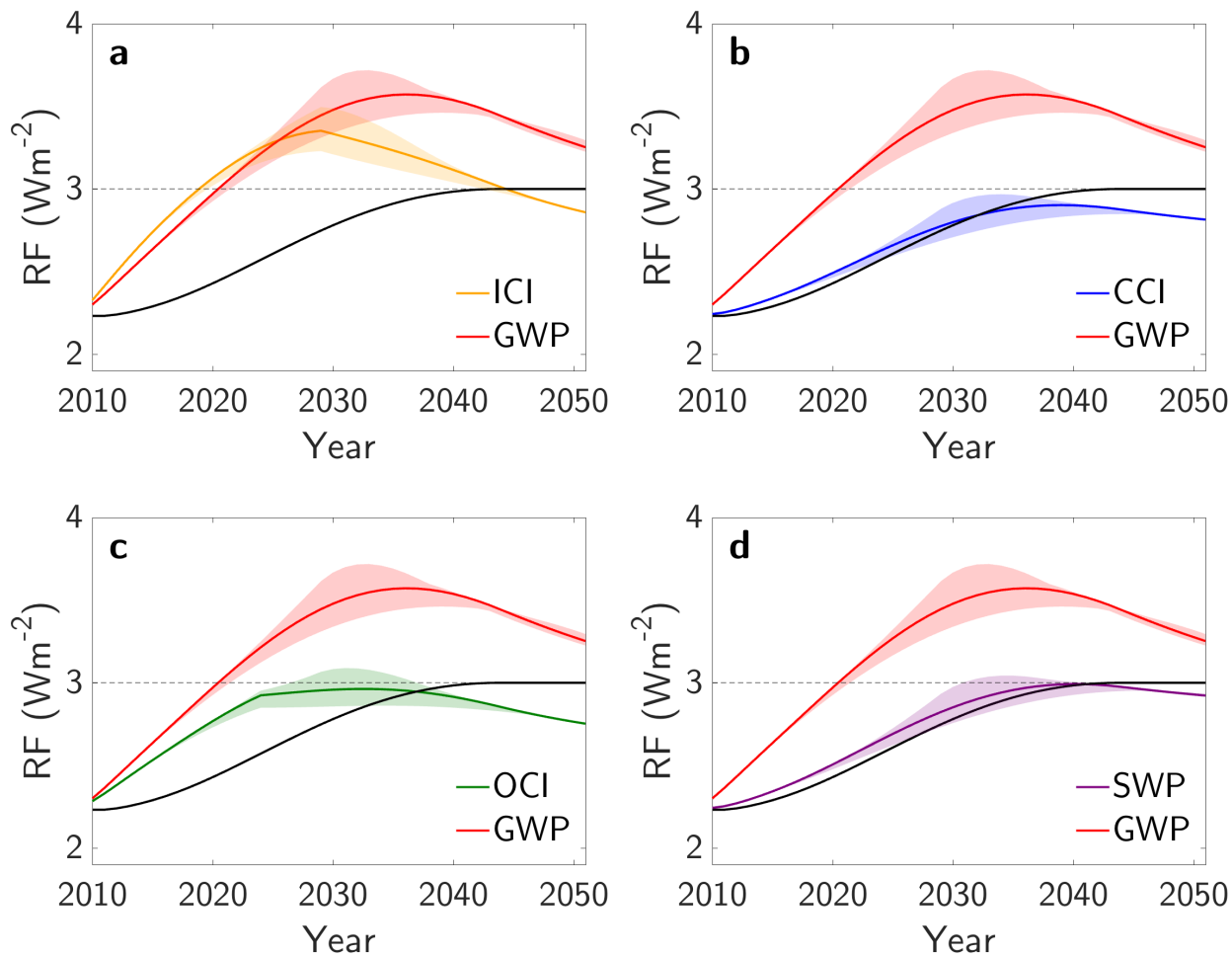

Figure 2: Radiative forcing outcomes under alternative equivalency metrics compared to the $\mathrm{GWP}$, for a $3 \mathrm{Wm}^{-2}$ stabilization target (a-d). The black line is the radiative forcing pathway that would result if all emissions were $\mathrm{CO}_{2}$. The solid colored lines show pathways that result when the expected emissions budget is realized using metrics to determine allowed emissions of both $\mathrm{CO}_{2}$ and $\mathrm{CH}_{4}$. Shaded regions show the range of outcomes across the scenarios tested. The alternative metrics exhibit lower overshoots, compared to the GWP.

overshoots. The CCI eliminates overshoots completely but creates the largest undershoots in later years. For $4.5 \mathrm{Wm}^{-2}$, in contrast, the GWP leads to smaller relative overshoots, and one of the alternative metrics, the ICI, leads to higher overshoots than the GWP. The OCI and SWP lead to small overshoots or undershoots, depending on the scenario, and the CCI leads to more significant undershoots than in the $3 \mathrm{Wm}^{-2}$ case. These results suggest that metric selection should take into account the climate policy goal, since one metric (the ICI) outperforms the GWP under one stabilization level but not the other.

Despite some differences, there are similarities in the radiative forcing overshoot reductions achieved, as compared to the GWP, for a large range of climate-goal-inspired metrics, including the static SWP and the dynamic CCI and OCI. This result is explained by the fact that emissions budgets must decrease dramatically over the next few decades to meet stabilization targets, particularly for the $3 \mathrm{Wm}^{-2}$ target and to a lesser extent for $4.5 \mathrm{Wm}^{-2}$ (see Sec. D). Consequently, metric values during this critical early period strongly determine the radiative forcing pathway, whereas metric values several decades in the future (when the values of the static and dynamic metrics begin to diverge) are relatively less important because emissions budgets are very low. If emissions are more significant in later years, the SWP leads to higher radiative forcing than the dynamic OCI and CCI. Fig. 5 shows a case where metrics are applied to a constant emissions budget set at today's level, and $30 \%$ of the budget is allocated to $\mathrm{CH}_{4}$. The analytical expression 

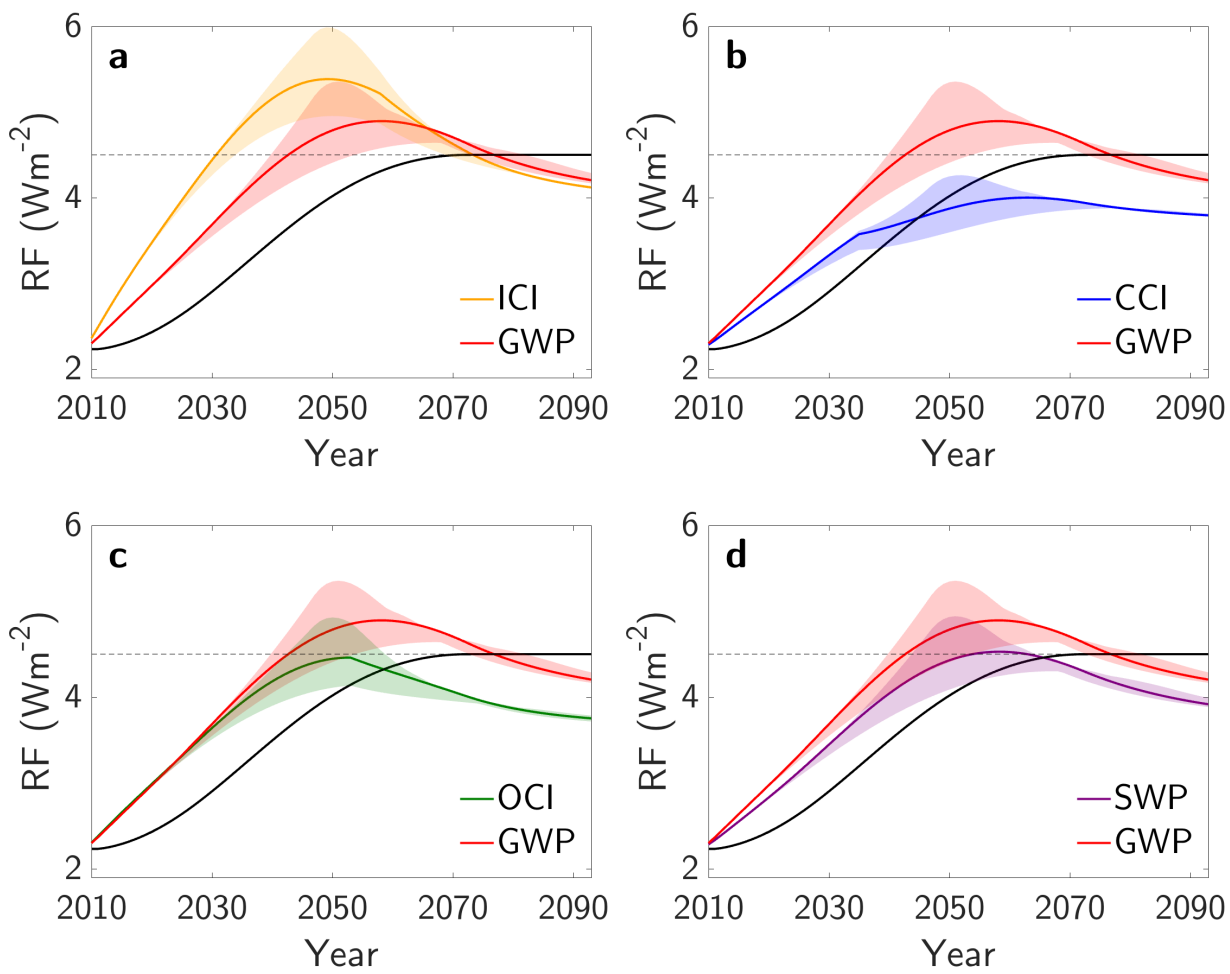

Figure 3: Radiative forcing outcomes under alternative equivalency metrics compared to the GWP, for a 4.5 Wm ${ }^{-2}$ stabilization target (a-d). The black line is the radiative forcing pathway that would result if all emissions were $\mathrm{CO}_{2}$. The solid colored lines show pathways that result when the expected emissions budget is realized using metrics to determine allowed emissions of both $\mathrm{CO}_{2}$ and $\mathrm{CH}_{4}$. Shaded regions show the range of outcomes across the scenarios tested. Compared with the GWP, the ICI leads to higher overshoots and the other metrics lead to lower or no overshoots. 
for radiative forcing performance, derived at the end of this section, provides further intuition for this result (see also Sec. B1).

The radiative forcing overshoots that may result from using a metric should be contextualized by the energy consumption that the metric permits. High metric values reduce the risk of overshoots but place more stringent restrictions on emissions and concurrent energy consumption levels, if the emissions intensity of energy does not keep up with the rate of emissions decline. We present a case in Fig. 4 where the emissions intensities of the portfolios decline by $1 \%$ annually across-the-board. This assumption impacts the total consumption estimated by our model but not the relative consumption allowed by different metrics, which depends only on the ratio of $\mathrm{CH}_{4}$ and $\mathrm{CO}_{2}$ emissions intensities. Consumption is determined by use of the portfolio with the lowest $\mathrm{CO}_{2}$-equivalent emissions per unit energy. With the GWP, a relatively small impact value is placed on $\mathrm{CH}_{4}$, and the high- $\mathrm{CH}_{4}$ portfolio attains the lower $\mathrm{CO}_{2}$-equivalent intensity. Since the GWP is constant, this portfolio is selected in all years. The SWP under a $4.5 \mathrm{Wm}^{-2}$ stabilization level also leads to the selection of the $\mathrm{CH}_{4}$-heavy portfolio, but under $3 \mathrm{Wm}^{-2}$ the $\mathrm{CH}_{4}$-light portfolio is selected, due to the closer stabilization time. The dynamic metrics generally lead to the selection of the $\mathrm{CH}_{4}$-heavy portfolio initially but switch to the $\mathrm{CH}_{4}$-light after some period of time, depending on the stringency of the metric. However, different energy consumption levels are permitted for each metric, depending on the metric value(s).

The results suggest that no metric is dominant in all situations: there are tradeoffs between economic and climate performance. For $3 \mathrm{Wm}^{-2}$, all alternative metrics examined reduce total energy consumption relative to the GWP and lead to correspondingly lower overshoots. However, the amount of reduction varies across metrics, and the ICI allows higher energy consumption than the GWP in early years. For $4.5 \mathrm{Wm}^{-2}$, the ICI allows higher total energy consumption than the GWP but leads to higher overshoots. However, certain alternative metrics can dramatically reduce overshoots while maintaining energy consumption at or above today's level for several decades, with only modest improvements in the emissions intensity of the portfolios.

We can also examine these results analytically to identify the drivers of metric performance. Intended radiative forcing $\mathrm{RF}_{\mathrm{I}}$, written in terms of budgeted emissions $e\left(t^{\prime}\right)$, is

$$
\mathrm{RF}_{\mathrm{I}}(t)=A_{\mathrm{K}} \int_{0}^{t} e\left(t^{\prime}\right) f_{\mathrm{K}}\left(t, t^{\prime}\right) d t^{\prime}+\mathrm{RF}_{\mathrm{KL}}(t)+\mathrm{RF}_{\mathrm{ML}}(t)+\mathrm{RF}_{\mathrm{N}}(t),
$$

where $A$ and $f$ are defined previously, $\mathrm{RF}_{\mathrm{KL}}$ and $\mathrm{RF}_{\mathrm{ML}}$ are the legacy radiative forcing impacts of historical $\mathrm{CO}_{2}$ and $\mathrm{CH}_{4}$ emissions, and $\mathrm{RF}_{\mathrm{N}}$ denotes radiative forcing from all non- $\mathrm{CO}_{2}$, non- $\mathrm{CH}_{4}$ sources. For simplicity, suppose a fixed fraction $q$ of $\mathrm{CO}_{2}$-equivalent emissions is allocated to $\mathrm{CH}_{4}$, with equivalence determined by the metric to be tested. Actual radiative forcing $\mathrm{RF}_{\mathrm{A}}$ would be

$$
\begin{aligned}
\mathrm{RF}_{\mathrm{A}}(t)=A_{\mathrm{K}}(1-q) \int_{0}^{t} e\left(t^{\prime}\right) f_{\mathrm{K}}\left(t, t^{\prime}\right) d t^{\prime} & +A_{\mathrm{M}} q \int_{0}^{t} \frac{e\left(t^{\prime}\right)}{\mu\left(t^{\prime}\right)} f_{\mathrm{M}}\left(t, t^{\prime}\right) d t^{\prime} \\
& +\mathrm{RF}_{\mathrm{KL}}(t)+\mathrm{RF}_{\mathrm{ML}}(t)+\mathrm{RF}_{\mathrm{N}}(t),
\end{aligned}
$$



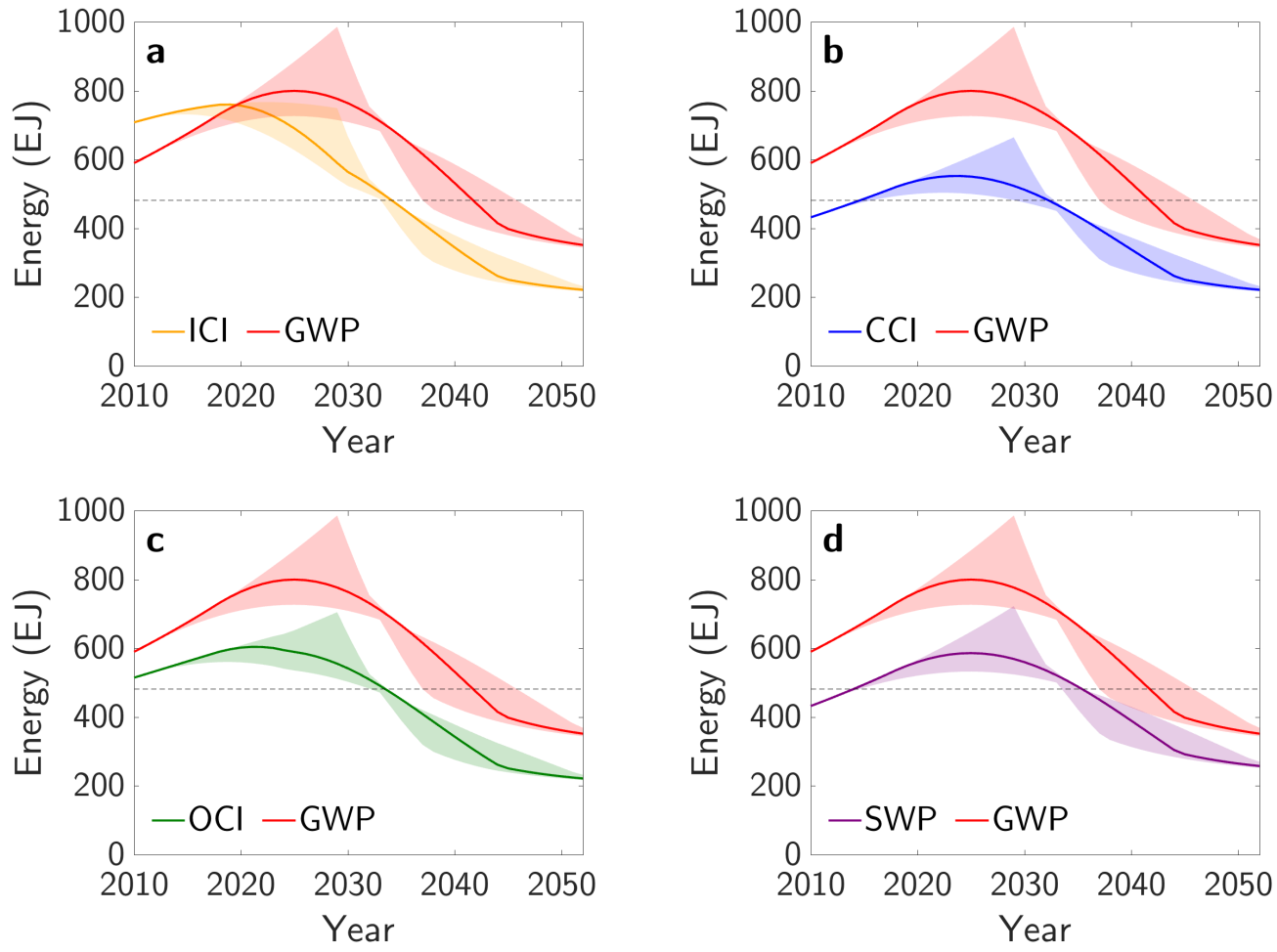

Figure 4: Energy consumption outcomes under alternative equivalency metrics compared to the GWP, for a 3 Wm ${ }^{-2}$ stabilization target (a-d). Dashed lines indicate today's consumption levels. Energy consumption trends are affected by the emissions intensities of energy technologies modeled. In the scenario modeled here, the emissions intensity of energy decreases by $1 \%$ per year; consumption levels would be higher if the emissions intensities of energy were lower. The solid colored lines show pathways that result when the expected emissions budget is realized, and shaded region show the range of outcomes across the scenarios tested. The GWP tends to allow more energy consumption, except in the case of the ICI metric in early years. Dynamic metrics tend to allow higher relative consumption early on and lower relative consumption later on, as compared to static metrics.

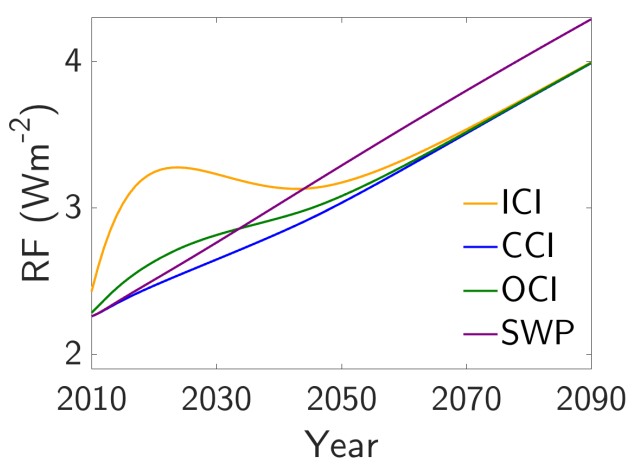

Figure 5: Radiative forcing outcomes under alternative static (SWP) and dynamic (ICI, CCI, OCI) metrics based on a 3 Wm ${ }^{-2}$ stabilization level, in a scenario in which global emissions continue at today's levels and metrics are applied to a constant $30 \%$ of total emissions. The ICI leads to higher radiative forcing values early on, but the performance of the three dynamic metrics converges later on at a lower radiative forcing value than the static $\mathrm{SWP}$, which places a lower impact value on $\mathrm{CH}_{4}$ than the dynamic metrics in later years. 


$$
\Delta \mathrm{RF}(t)=q \int_{0}^{t} A_{\mathrm{M}} e\left(t^{\prime}\right) f_{\mathrm{M}}\left(t, t^{\prime}\right)\left[\frac{1}{\mu\left(t^{\prime}\right)}-\frac{A_{\mathrm{K}} f_{\mathrm{K}}\left(t, t^{\prime}\right)}{A_{\mathrm{M}} f_{\mathrm{M}}\left(t, t^{\prime}\right)}\right] d t^{\prime} .
$$

This formulation of technology selection is analogous to the testing simulation in Fig. 5 but differs from that in Figs. 2-4, where gases are emitted in given mass ratios by each technology. However, it provides intuition about metric performance in both situations. In general, $\Delta \mathrm{RF}$ is proportional to the fraction $q$ of emissions allocated to $\mathrm{CH}_{4}$. The contribution to $\Delta \mathrm{RF}$ specifically due to $\mathrm{CH}_{4}$ emissions in a prior year $t^{\prime}$ is proportional to the difference between the metric valuation $\left(\mu^{-1}\right)$ and the "correct" valuation $\left(\left(A_{K} f_{K}\right) /\left(A_{M} f_{M}\right)\right)$ of $\mathrm{CH}_{4}$ emissions from that year.

Overshoots are closely related to $\Delta \mathrm{RF}$; they occur when $\Delta \mathrm{RF}$ is sufficiently positive and the intended pathway $\mathrm{RF}_{\mathrm{I}}$ is close to the stabilization level. The alternative metrics we examine place a different impact value on $\mathrm{CH}_{4}$ depending on the intended stabilization time $t_{\mathrm{S}}$, which is connected to the stabilization level: stabilization may occur earlier for lower targets. Additionally, dynamic metrics change the value they place on $\mathrm{CH}_{4}$ depending on the proximity to the year $t_{\mathrm{S}}$, causing the metric to stay more closely related to the "correct" value $\left(A_{K} f_{K}\right) /\left(A_{M} f_{M}\right)$ near those impact years where overshoot is most likely to occur. The highest risk of overshoot occurs when $t_{\mathrm{S}}$ is assumed to be a later value when constructing the metric (causing $\mu^{-1}$ to be large early on), but the emissions budget stabilizes earlier. Conversely, the risks of overshoots are small for selecting a $t_{\mathrm{S}}$ that is too early (Sec. A4). Errors may also cancel out over time if an overly high impact value is assigned to $\mathrm{CH}_{4}$ early on but a too low impact value is assigned later on.

\section{Discussion and Conclusion}

This paper presents an approach for testing emissions equivalency metrics against climate policy goals, to

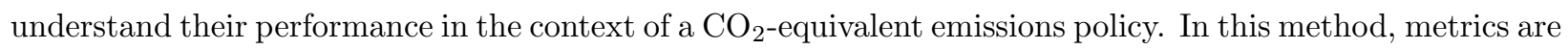
used to evaluate the $\mathrm{CO}_{2}$-equivalent emissions intensities of technologies which are then used in compliance with an emissions cap. The method we develop introduces two main features necessary to test metrics in a practical context: (1) actual climate outcomes are not constrained artificially and (2) technology choice is explicitly modeled, which bounds the potential error due to high $\mathrm{CH}_{4}$ emissions.

Our tests focus on comparing alternative radiative forcing metrics to the GWP. The results suggest that, if chosen carefully, alternative metrics that incorporate information on an intended climate goal show improved performance in terms of avoiding a radiative forcing overshoot. Importantly, these goal-inspired metrics can perform well despite uncertainty in the timing of emissions mitigation. The appropriate metric depends on the radiative forcing stabilization target (a policy target) and resulting estimated timelines for stabilization. With longer timelines, stabilization-time-based metrics take on lower values, and less conservative metrics like the ICI may lead to higher radiative forcing overshoots than the GWP. We also find that dynamic and static metrics lead to different technology selection behavior. The dynamic metrics can lead to technology switching, where higher- $\mathrm{CH}_{4}$ technologies are followed by lower ones, thereby permitting more 

in early years can be important because it can allow for time to develop and increase the adoption of lower emissions energy technologies while meeting energy demand in the near-term.

The shape of the simple ICI and CCI resembles that of the complex OCI, whose values fall in-between those of the ICI and CCI prior to stabilization. While similar in shape, there may be advantages to using simpler metrics over more complex ones. Simple metrics like the ICI and CCI have transparent, closed-form expressions. The OCI, in contrast, depends on the output of an optimization model, which requires a variety of input assumptions that may not be transparent to, and can differ across, users. The choice between the ICI, CCI, or another simple, closed-form weighting scheme requires considering tradeoffs between climate risks (e.g. overshoots of intended stabilization levels) and economic costs (e.g higher-than-required reductions in energy consumption). The nature of these tradeoffs depends on the intended climate policy goal. The static SWP is even simpler, as its values do not change over time, and shows similar performance to the CCI and OCI under emissions reduction policies, although long-term radiative forcing outcomes may diverge under prolonged high emissions.

The method presented here can be extended to test metrics based on temperature or economic impacts. There are challenges to basing metrics on temperature, including uncertainty in the temperature sensitivity and a wide range of possible stabilization years (and even definitions of stabilization). Economic metrics require further assumptions, introducing additional uncertainty. At the same time, dynamic metrics based on these impacts tend to have similar shapes to ones based on radiative forcing [14, 24, 28, 38], and some of the insights on metric performance described here may transcend the measure of impact used. This suggests a pragmatic approach to metric selection, by choosing metrics that are easier to compute without necessarily incurring worse outcomes. A testing-focused approach can also guide new metric formulations.

Greenhouse gas equivalency metrics are widely used in a variety of applications, including technology evaluation [7, 53] and emissions trading. They can be used to optimize sectoral energy consumption[36] or set $\mathrm{CH}_{4}$ mitigation targets to accompany $\mathrm{CO}_{2}$-focused legislation [27]. Current policies, including new emissions standards for power plants in the U.S. [46, 47] (focused on $\mathrm{CO}_{2}$ ) and regulations addressing $\mathrm{CH}_{4}$ [48], both depend on implicit or explicit comparisons of gases, as do national commitments referenced in the Paris climate agreement [45]. Even under a climate policy that regulates gases (or baskets of similar gases) separately [40], comparisons of gases are needed to allocate emissions budgets and set emissions reduction targets. Equivalency metrics present one approach that is widely used in practice for making these comparisons. For the policy context considered here, while no metric performs perfectly when tested, we find that alternative metrics that are based on a radiative forcing stabilization goal can exhibit performance improvements over the GWP when selecting energy technologies to meet mitigation goals. 


\section{Acknowledgements}

We gratefully acknowledge support from the MIT Energy Initiative, the Leading Technology Policy Initiative, the Charles E. Reed Faculty Initiatives Fund, and the New England University Transportation Center under DOT Grant No. DTRT12-G-UTC01. MRE acknowledges support from a National Science Foundation Graduate Research Fellowship Program under Grant No. 1122374. We also thank Mandira Roy and Victor Ocaña for helpful discussions regarding this research.

\section{Biographies}

Morgan R. Edwards is a Ph.D. candidate at the Institute for Data, Systems, and Society at MIT. Her dissertation focuses on the climate impacts of mitigating short-lived greenhouse gas emissions and implications for technology evaluation. Before coming to MIT, she worked on alternative energy and energy efficiency projects at the University of North Carolina at Chapel Hill, the Higher School of Economics in Russia, and King Mongkut's University of Technology Thonburi in Thailand.

James McNerney is a postdoctoral associate at the Institute for Data, Systems, and Society (IDSS) at MIT. Formerly he was a graduate fellow at the Santa Fe Institute and Boston University, where he completed his Ph.D. in physics on the topic of cost evolution of technologies. His current work focuses on technology evaluation and technology evolution at both the small scale of individual technologies and the large scale of whole economies.

Jessika E. Trancik is the Atlantic Richfield Associate Professor in Energy Studies at the Institute for Data, Systems and Society at the Massachusetts Institute of Technology. She is also an external professor at the Santa Fe Institute. She received her B.S. from Cornell University and her Ph.D. from the University of Oxford, and spent several years at the Santa Fe Institute as an Omidyar Fellow, and at Columbia University as an Earth Institute Fellow. Her research focuses on evaluating the economic and environmental impacts of energy technologies against climate change mitigation goals, to inform the development of technologies and policy.

[1] Kyoto Protocol to the United Nations Framework Convention on Climate Change, FCCC/CP/1997/7/Add.1, 37 I.L.M., December 1998.

[2] M. R. Allen, D. J. Frame, C. Huntingford, C. D. Jones, J. A. Lowe, M. Meinshausen, and N. Meinshausen. Warming caused by cumulative carbon emissions towards the trillionth tonne. Nature, 458(7242):1163-1166, 2009.

[3] M. R. Allen, J. S. Fuglestvedt, K. P. Shine, A. Reisinger, R. T. Pierrehumbert, and P. M. Forster. New use of global warming potentials to compare cumulative and short-lived climate pollutants. Nature Clim. Change, 2016. 
[4] R. A. Alvarez, S. W. Pacala, J. J. Winebrake, W. L. Chameides, and S. P. Hamburg. Greater focus needed on methane leakage from natural gas infrastructure. Proc. Natl Acad. Sci USA, 109(17):6435$6440,2012$.

[5] C. Azar and D. J. A. Johansson. Valuing the non- $\mathrm{CO}_{2}$ climate impacts of aviation. Climatic Change, 111(3-4):559-579, 2011.

[6] O. Boucher. Comparison of physically- and economically-based $\mathrm{CO}_{2}$-equivalencies for methane. Earth Syst. Dynam., 3(1):49-61, 2012.

[7] A. R. Brandt, G. A. Heath, E. A. Kort, F. O. O'Sullivan, G. Petron, S. M. Jordaan, P. Tans, J. Wilcox, A. M. Gopstein, D. Arent, S. Wofsy, N. J. Brown, R. Bradley, G. D. Stucky, D. Eardley, and R. Harriss. Methane leaks from North American natural gas systems. Science, 343:733-735, 2014.

[8] M. E. Brennan and B. F. Zaitchik. On the potential for alternative greenhouse gas equivalence metrics to influence sectoral mitigation patterns. Environ. Res. Lett., 8(1), 2013.

[9] J. R. Camuzeaux, R. A. Alvarez, S. A. Brooks, J. B. Browne, and T. Sterner. Influence of methane emissions and vehicle efficiency on the climate implications of heavy-duty natural gas trucks. Environ. Sci. Technol., 49(11):6402-6410, 2015.

[10] F. Cherubini and A. H. Strømman. Life cycle assessment of bioenergy systems: State of the art and future challenges. Bioresource Technol., 102(2):437-451, 2011.

[11] J. S. Daniel, S. Solomon, T. J. Sanford, M. McFarland, J. S. Fuglestvedt, and P. Friedlingstein. Limitations of single-basket trading: Lessons from the Montreal Protocol for climate policy. Climatic Change, 111(2):241-248, 2011.

[12] O. Deuber, G. Luderer, and O. Edenhofer. Physico-economic evaluation of climate metrics: A conceptual framework. Environ. Sci. Policy, 9:37-45, 2013.

[13] O. Edenhofer, R. Pichs-Madruga, Y. Sokona, J. C. Minx, E. Farahani, S. Kadner, K. Seyboth, A. Adler, I. Baum, S. Brunner, P. Eickemeier, B. Kriemann, J. Savolainen, S. Schl omer, C. von Stechow, and T. Zwickel, editors. Climate Change 2014: Mitigation of Climate Change. Intergovernmental Panel on Climate Change, Cambridge University Press, 2014.

[14] M. R. Edwards and J. E. Trancik. Climate impacts of energy technologies depend on emissions timing. Nature Clim. Change, 4(5):347-352, 2014.

[15] T. Ekholm, T. J. Lindroos, and I. Savolainen. Robustness of climate metrics under climate policy ambiguity. Environ. Sci. Policy, 31:44-52, 2013.

[16] E. D. Frank, J. Han, I. Palou-Rivera, A. Elgowainy, and M. Q. Wang. Methane and nitrous oxide emissions affect the life-cycle analysis of algal biofuels. Environ. Res. Lett., 7(1):014030, 2012. 
[17] J. Fuglestvedt, T. Berntsen, G. Myhre, K. Rypdal, and R. B. Skeie. Climate forcing from the transport sectors. Proc. Natl. Acad. Sci. USA, 105(2):454-458, 2008.

[18] J. S. Fuglestvedt, T. K. Berntsen, O. Godal, R. Sausen, K. P. Shine, and T. Skodvin. Metrics of climate change: Assessing radiative forcing and emission indices. Climatic Change, 58:267-331, 2003.

[19] J. S. Fuglestvedt, K. P. Shine, T. Berntsen, J. Cook, D. S. Lee, A. Stenke, R. B. Skeie, G. J. M. Velders, and I. A. Waitz. Transport impacts on atmosphere and climate: Metrics. Atmos. Environ., 44(37):4648-4677, 2010.

[20] G. A. Heath, P. O'Donoughue, D. J. Arent, and M. Bazilian. Harmonization of initial estimates of shale gas life cycle greenhouse gas emissions for electric power generation. Proc. Natl. Acad. Sci. USA, 111(31):E3167-E3176, 2014.

[21] B. Hong and R. W. Howarth. Greenhouse gas emissions from domestic hot water: heat pumps compared to most commonly used systems. Energy Sci. Eng., 2016.

[22] J. T. Houghton, G. J. Jenkins, and J. J. Ephraums, editors. Climate Change: The IPCC Scientific Assessment. Intergovernmental Panel on Climate Change (IPCC), Cambridge University Press, 1990.

[23] C. Huntingford, J. A. Lowe, N. Howarth, N. H. A. Bowerman, L. K. Gohar, A. Otto, D. S. Lee, S. M. Smith, M. G. J. den Elzen, and D. P. van Vuuren. The implications of carbon dioxide and methane exchange for the heavy mitigation rcp2.6 scenario under two metrics. Environ. Sci. Policy, 51:77-87, 2015.

[24] D. J. A. Johansson. Economics- and physical-based metrics for comparing greenhouse gases. Climatic Change, 110(1-2):123-141, 2012.

[25] D. J. A. Johansson, U. M. Persson, and C. Azar. The cost of using global warming potentials: Analysing the trade off between $\mathrm{CO}_{2}, \mathrm{CH}_{4}$ and $\mathrm{N}_{2} \mathrm{O}$. Climatic Change, 77(3-4):291-309, 2006.

[26] M. Kandlikar. Indices for comparing greenhouse gas emissions: Integrating science and economics. Energ. Econ., 18:265-281, 1996.

[27] M. M. Klemun and J. E Trancik. Methane impacts of $\mathrm{CO}_{2}$-focused energy policies. In Review.

[28] A. S. Manne and R. G. Richels. An alternative approach to establishing trade-offs among greenhouse gases. Nature, 410(6829):675-677, 2001.

[29] A. L. Marten and S. C. Newbold. Estimating the social cost of non- $\mathrm{CO}_{2}$ GHG emissions: Methane and nitrous oxide. Energy Policy, 51:957-972, 2012.

[30] B. C. O'Neill. The jury is still out on global warming potentials. Climatic Change, 44:427-443, 2000. 
[31] B. C. O'Neill. Economics, natural science, and the costs of global warming potentials. Climatic Change, 58:251-260, 2003.

[32] G. P. Peters, B. Aamaas, T. Berntsen, and J. S Fuglestvedt. The integrated global temperature change potential (iGTP) and relationships between emission metrics. Environ. Res. Lett., 6(4), 2011.

[33] J. M. Reilly and K. R. Richards. Climate change damage and the trace gas index issue. Environ. Resour. Econ., 3:41-61, 1993.

[34] A. Reisinger, P. Havlik, K. Riahi, O. van Vliet, M. Obersteiner, and M. Herrero. Implications of alternative metrics for global mitigation costs and greenhouse gas emissions from agriculture. Climatic Change, 117(4):677-690, 2013.

[35] G. H. Roe and M. B. Baker. Why is climate sensitivity so unpredictable? Science, 318:629-632, 2007.

[36] M. Roy, M. R. Edwards, and J. E. Trancik. Methane mitigation timelines to inform energy technology evaluation. Environ. Res. Lett., 10:114024, 2015.

[37] K. P. Shine. The global warming potential - the need for an interdisciplinary retrial. Climatic Change, 96(4):467-472, 2009.

[38] K. P. Shine, T. K. Berntsen, J. S. Fuglestvedt, R. B. Skeie, and N. Stuber. Comparing the climate effect of emissions of short- and long-lived climate agents. Phil. Trans. R. Soc. A, 365(1856):1903-1914, 2007.

[39] K. P. Shine, J. S. Fuglestvedt, K. Hailemariam, and N. Stuber. Alternatives to the global warming potential for comparing climate impacts of emissions of greenhouse gases. Climatic Change, 68(3):281$302,2005$.

[40] S. M. Smith, J. A. Lowe, N. H. A. Bowerman, L. K. Gohar, C. Huntingford, and M. R. Allen. Equivalence of greenhouse-gas emissions for peak temperature limits. Nature Clim. Change, 2(11):8-11, 2012.

[41] T. F. Stocker, D. Qin, G.-K. Plattner, M. Tignor, S. K. Allen, J. Boschung, A. Nauels, Y. Xia, V. Bex, and P. M. Midgley, editors. Climate Change 2013: The Physical Science Basis. Intergovernmental Panel on Climate Change, Cambridge University Press, 2013.

[42] K. Tanaka, D. J. A. Johansson, B. C. O’Neill, and J. S. Fuglestvedt. Emissions metrics under a $2^{\circ}$ C stabilization target. Climatic Change, 117(4):933-941, 2013.

[43] K. Tanaka, B. C. O’Neill, D. Rokityanskiy, M. Obersteiner, and R. S. J. Tol. Evaluating global warming potentials with historical temperature. Climatic Change, 96:443-466, 2009.

[44] R. S. J. Tol, T. K. Berntsen, B. C. O’Neill, J. S. Fuglestvedt, and K. P. Shine. A unifying framework for metrics for aggregating the climate effect of different emissions. Environ. Res. Lett., 7, 2012. 
[45] United Nations Framework Convention on Climate Change. Adoption of the Paris Agreement, Proposal by the President, 2015.

[46] U.S. Environmental Protection Agency. Carbon pollution emission guidelines for existing stationary sources: Electric utility generation units. Federal Register, 80:64661-65120, 2015.

[47] U.S. Environmental Protection Agency. Standards of performance for greenhouse gas emissions from new, modified, and reconstructed stationary sources: Electric utility generating units. Federal Register, 80:64510-64660, 2015.

[48] U.S. Environmental Protection Agency. Oil and natural gas sector: Emissions standards for new, reconstructed, and modified sources. Final Rule, EPA-HQ-OAR-2010-0505, 2016.

[49] M. van den Berg, A. F. Hof, J. van Vliet, and D. P. van Vuuren. Impact of the choice of emission metric on greenhouse gas abatement and costs. Environ. Res. Lett., 10(2):24001, 2015.

[50] D. P. van Vuuren, J. Edmonds, M. Kainuma, K. Riahi, A. Thomson, K. Hibbard, G. C. Hurtt, T. Kram, V. Krey, J. F. Lamarque, T. Masui, M. Meinshausen, N. Nakicenovic, S. J. Smith, and S. K. Rose. The representative concentration pathways: An overview. Climatic Change, 109(1):5-31, 2011.

[51] D. P. van Vuuren, B. Eickhout, P. L. Lucas, and M. G. J. den Elzen. Long-term multi-gas scenarios to stabilise radiative forcing: Exploring costs and benefits within an integrated assessment framework. Energy J., 27:201-234, 2006.

[52] D. P. van Vuuren, J. Weyant, and F. de la Chesnaye. Multi-gas scenarios to stabilize radiative forcing. Energ. Econ., 28(1):102-120, 2006.

[53] D. Weisser. A guide to life-cycle greenhouse gas (GHG) emissions from electric supply technologies. Energy, 32(9):1543-1559, 2007. 